\title{
Composite analysis of immunological and metabolic markers defines novel subtypes of triple negative breast cancer
}

Thomas A Adams ${ }^{1}$, Paris J Vail ${ }^{2}$, Amanda Ruiz ${ }^{2}$, Mehri Mollaee ${ }^{3}$, Peter A McCue ${ }^{3}$, Erik S Knudsen ${ }^{2}$ and Agnieszka K Witkiewicz ${ }^{1,2}$

${ }^{1}$ Department of Pathology, University of Arizona, Tucson, AZ, USA; ${ }^{2}$ University of Arizona Cancer Center, Tucson, AZ, USA and ${ }^{3}$ Department of Pathology, Thomas Jefferson University, Philadelphia, PA, USA

\begin{abstract}
Cancer biology is influenced by the tumor microenvironment, which impacts disease prognosis and therapeutic interventions. The inter-relationship of tumor-infiltrating lymphocytes, immune response regulators, and a glycolytic tumor environment was evaluated in a cohort of 183 largely consecutive patients with triple negative breast cancer diagnosis. High levels of tumor-infiltrating lymphocytes were associated with improved survival of triple negative breast cancer cases. However, elevated levels of PD-L1, CD163, and FOXP3 were individually associated with significantly decreased overall survival. These three determinants were significantly correlated, and could serve to differentiate the prognostic significance of tumor-infiltrating lymphocytes. Interestingly, a glycolytic tumor environment, as determined by the expression of MCT4 in the tumor stroma, was associated with the immune evasive environment and poor prognosis. Clustering of all markers defined four distinct triple negative breast cancer subtypes that harbored prognostic significance in multivariate analysis. Immune and metabolic markers stratified triple negative breast cancer into subtypes that have prognostic significance and implications for therapies targeting immune checkpoints and tumor metabolism.

Modern Pathology (2018) 31, 288-298; doi:10.1038/modpathol.2017.126; published online 6 October 2017
\end{abstract}

Triple negative breast cancer accounts for $\sim 15 \%$ of breast cancers and is defined by a lack of expression of estrogen receptor (ER), progesterone receptor (PR), and HER2/neu. ${ }^{1}$ Triple negative breast cancer affects younger patients ( $<50$ years), is more prevalent in African-American women, and is more aggressive than breast tumors of other molecular subtypes. ${ }^{1-8}$ In contrast to ER-positive or HER2-positive breast carcinomas, there are no approved molecularly targeted therapies for triple negative breast cancer. ${ }^{9-11}$ As a result, triple negative breast cancer treatment has remained dependent on the efficacy of conventional chemotherapy, radiation, and surgery. Previous studies have indicated that tumor-infiltrating lymphocytes are over-represented in triple negative breast cancers compared with other breast cancer subtypes. ${ }^{12-14}$ Given the success of immunotherapeutic agents in melanoma and lung cancer, ${ }^{15,16}$

Correspondence: Professor AK Witkiewicz, Cancer Center, University of Arizona Cancer Center, 1515 N Campbell Avenue, 4963, Tucson, AZ 85724, USA.

E-mail: awitki@email.arizona.edu

Received 13 July 2017; revised 1 August 2017; accepted 3 August 2017; published online 6 October 2017 there is growing interest in the potential for immunotherapeutic strategies to treat breast cancer. It has been hypothesized that these strategies would be effective in patients whose tumors have a brisk lymphocytic infiltrate. ${ }^{12,17}$ The International TumorInfiltrating Lymphocytes Working Group 2014 provided a framework for enumerating tumorinfiltrating lymphocytes, and subsequent work has shown agreement between pathologists in the use of these recommendations to define significant tumorinfiltrating lymphocytes. ${ }^{12,18}$ The degree of lymphocytic infiltration assessed by routine histology has been shown to have predictive and prognostic value in triple negative breast cancer. ${ }^{18}$

Tumor biology is influenced by the intrinsic microenvironment and the host immune response. The development of immune checkpoint inhibitors provides an opportunity to augment the antitumor immune response that has proved effective in multiple clinical studies. PD-1 is a checkpoint molecule in the immune response and is expressed on T cells. ${ }^{19}$ PD-L1, a ligand for PD-1, is reported to be expressed on triple negative breast cancer cells as well as infiltrating lymphocytes and thought to be a mechanism for evading the immune response to 
tumor-specific $\mathrm{T}$ cells. ${ }^{19,20}$ Immune modulatory agents, such as PD-1 and PD-L1 inhibitors, have shown activity in melanoma and bladder cancer. Recent studies involving triple negative breast cancer have shown similar patterns of response and adverse events as described in melanoma. ${ }^{21-23}$

Importantly in tumors, there are other determinants of tumor-associated immune suppression including regulatory $\mathrm{T}$ cells, which express FOXP3, and M2 macrophages, marked by CD163. ${ }^{24,25}$ Regulatory $\mathrm{T}$ cells, a unique subset of CD4+ helper $\mathrm{T}$ cells of the CD4+/CD25+ phenotype, can suppress proliferation and cytokine secretion of effector T lymphocytes through immunoregulation. ${ }^{24}$ The most specific and reliable marker of regulatory $\mathrm{T}$ cells is FOXP3, and elevated FOXP3 lymphocytes are thought to contribute to tumor immune evasion in breast cancer. ${ }^{24}$ Tumor-associated macrophages are alternatively activated macrophages that enhance tumor progression by promoting tumor cell invasion, migration, and angiogenesis. $^{25}$ Tumor-associated macrophages have an anti-inflammatory function similar to M2 macrophages. Expression of the CD163 antigen is a highly specific marker for M2 macrophages. ${ }^{25}$

The nature of the immune infiltrate is believed to be related to both the innate immunogenicity of the tumor and the tumor microenvironment. Neo-epitopes and the presentation of non-self antigens by the tumor represents one of the key drivers of antitumor immune response. ${ }^{26,27}$ However, it is becoming increasingly clear that tumors can develop an environmental niche that influences the ability of lymphocytes to reach their targets and modulate the immune infiltrate. ${ }^{28}$ In particular, the metabolic state of a tumor can influence immune engagement.

Table 1 Clinicopathological features of the TNBC cohort

\begin{tabular}{lcc}
\hline Variable & $\mathrm{N}$ & Values \\
\hline Age & 180 & $55.5 \pm 13.7$ \\
Race & 179 & \\
$\quad$ White & & $76 \%(136)$ \\
$\quad$ African-American & & $24 \%(43)$ \\
Tumor size (cm) & 164 & $2.34 \pm 1.80$ \\
$\quad$ & 169 & \\
Histologic grade & & $75 \%(126)$ \\
$\quad 1-2$ & & $25 \%(43)$ \\
3 & 170 & $36 \%(62)$ \\
Stage & & $46 \%(78)$ \\
1 & & $12 \%(21)$ \\
2 & & $5 \%(9)$ \\
3 & & \\
4 & 144 & $59 \%(85)$ \\
Lymph node status & & $41 \%(59)$ \\
$\quad$ Negative & & $86.3 \pm 52.1$ \\
$\quad$ Positive & 174 &
\end{tabular}

Numbers in brackets are frequencies. $N$ denotes number of nonmissing observations.
A subset of tumor cells preferentially metabolize pyruvate into lactic acid regardless of the oxygen concentration. ${ }^{29}$ To export metabolic waste products, rapidly growing tumor cells overexpress proteins to allow for extrusion of products such as carbonic anhydrases and monocarboxylate transporters (MCTs). ${ }^{29-31}$ Interestingly, triple negative breast cancers were the most glycolytic of the breast tumors in a study of glycolytic markers that included MCT4. ${ }^{29}$ MCT4 was of prognostic significance and was associated with decreased overall survival. ${ }^{29,32}$ Inhibitors targeting glycolysis and monocarboxylate transporters are currently in pharmacologic development. ${ }^{29}$ In the present study, we investigated the relationship between immune response regulators (PD-L1, FOXP3, and CD163), glycolytic metabolism (MCT4), tumor-infiltrating lymphocytes, and their association with survival in triple negative breast cancers.

\section{Materials and methods}

\section{Study Population and Tumor Microarray Construction}

Study cases were obtained from surgical pathology files at Thomas Jefferson University with Institutional Review Board approval. The tissue microarrays were prepared from 180 largely consecutive patients with triple negative breast cancer. Clinical and treatment information was extracted by chart review. Clinicopathologic data included age, race, tumor size, stage, histologic grade, lymph node status, and overall survival (see Table 1). Treatment information could be obtained for 108 patients. For inclusion in this study as triple negative breast cancer, expressions of estrogen and progesterone receptors were not detected or were present in $<1 \%$ of tumor cells, with a satisfactory positive control. HER2 was scored $0-1+$ or 2+, and an absence of HER2 amplification by fluorescent in situ hybridization was required for negativity. Clinical and pathological variables were determined following well-established criteria. All triple negative breast cancer were graded according to the Elston-Ellis method; lymphovascular invasion was classified as either present or absent. The tumor tissue microarrays were constructed using a tissue arrayer (Veridiam). Two tissue cores $(0.6 \mu \mathrm{m}$ diameter) were sampled from each block to account for tumor and tissue heterogeneity and transferred to the recipient block.

\section{Immunostaining}

Immunohistochemistry was performed as described previously ${ }^{32,33}$ on 4-mm tissue microarrays sections using a standard avidin-biotin immunoperoxidase method with antibodies specific for CD163 (Cell Marque, prediluted), FOXP3 (Abcam, dilution 1:200), PD-L1 (Cell Signaling, dilution 1:600), and 
MCT4 (Santa Cruz, dilution 1:250). All stains were validated using recommended tissue controls, and evaluated for correct compartment specific staining by pathologists with experience in evaluating the indicated markers. Staining was performed on either a Dako or Ventana Benchmark automated immunohistochemical stainers.

\section{Tissue Microarrays Scoring and Statistical Analysis}

After staining, positive cells were counted and converted to a percentage of the total counted cells in a given field and expression of all examined markers was categorized as low or high based on the median percentage of positive cells. Stromal MCT4 staining was scored semi-quantitatively as negative (0, no staining), weak ( 1 , either diffuse weak staining or strong staining in $<30 \%$ of stromal cells per core), or strong (2, defined as strong staining of $30 \%$ or more of the stromal cells). Data were spot-checked for consensus and entered into a relational database by a third-party not affiliated with the study. Correlation between markers was obtained using the Spearman correlation method. Kaplan-Meier curves for both independent as well as combined markers were obtained using the survival package in $\mathrm{R}$ statistical software. ${ }^{34}$ Statistical significance and hazard ratios along with 95\% confidence intervals for the Kaplan-Meier curves was established using the log-rank $P$-value obtained from Cox proportional hazard regression analysis. A K-means clustering analysis was used for composite analysis of immune markers using $\mathrm{R}$ statistical software.

\section{Tumor-Infiltrating Lymphocytes}

Tumor-infiltrating lymphocytes were assessed based on criteria developed by International Tumor-Infiltrating Lymphocytes Working Group. ${ }^{18}$ Full face hematoxyln stained sections of the blocks used for the tissue microarrays construction were evaluated by a single pathologist. Tumor-infiltrating lymphocytes were assessed as $<5 \%$ or to the closest $5 \%$ increment. It has been previously reported that many studies evaluating the prognostic significance of tumor-infiltrating lymphocytes have used a cutoff of $50-60 \%$ to define lymphocyte predominant breast cancer. $^{12}$ In this study, $0-10 \%$ stromal tumorinfiltrating lymphocytes were scored as $0,10-50 \%$ as 1 , and $>50 \%$ as 2 .

\section{Results}

Tumor-Infiltrating Lymphocytes in Triple Negative Breast Cancer are Associated with Improved Survival

The degree of immune response is a key aspect of the tumor immune milieu and was characterized by enumerating tumor-infiltrating lymphocytes.
Stromal tumor-infiltrating lymphocytes were evaluated on full face hematoxylin and eosin-stained sections from the blocks used for tissue microarrays construction by a pathologist using established scoring criteria. ${ }^{35}$ This triple negative breast cancer cohort is derived from patients who underwent surgical resection in the absence of neoadjuvant therapy (Table 1). A diverse density of tumorinfiltrating lymphocytes was observed in the tissues as illustrated by the representative micrographs (Figure 1a). A subset of tumors was largely devoid of lymphocytic infiltrate in the stroma. Others exhibited intermediate or high numbers of tumorinfiltrating lymphocytes. The density of tumor-infiltrating lymphocytes was evaluated for association with survival. High tumor-infiltrating lymphocytes in the local tumor environment were associated with improved survival versus low tumor-infiltrating lymphocytes based on Kaplan-Meier analysis. The improved survival was most pronounced comparing low (score 0) versus high levels of tumor-infiltrating lymphocytes (score 2) (Figure 1b, left), but it was also observed when comparing low levels versus intermediate and high levels (score 0 vs 1, 2) (Figure 1b, right).

\section{Multiple Immune-Suppressive Mechanisms are Observed in Triple Negative Breast Cancer}

Expression of differing immune effectors within the tumor environment may reflect distinct mechanisms of immune suppression and have relevance to the utilization of immunotherapy in triple negative breast cancer.

Staining for PD-L1 showed substantial variance in the relative number of cells staining positively among triple negative breast cancer cases (Figure 2a). In univariate analysis, elevated levels of PD-L1 staining in the triple negative breast cancer tumor cells were associated with significantly decreased overall survival (Figure 2a), suggesting that suppression of immune function is associated with poor prognosis in triple negative breast cancer. FOXP3 staining highlighted regulatory T-cell lymphocytes, and elevated levels of FOXP3 staining were associated with significantly decreased overall survival by Kaplan-Meier analysis (Figure 2b). CD163 staining highlighted macrophages in the tumor cellular environment was also associated with significantly decreased overall survival by Kaplan-Meier analysis (Figure 2c).

That each of the immune-suppressive markers was associated with poor prognosis suggests that they may be correlated in the tumor cases. Spearman correlation analysis demonstrated that there was a high level of correlation between CD163, PD-L1, and FOXP3 $(P<0.001$ for all) (Figure 3a). In contrast, the correlation with tumor-infiltrating lymphocytes was not significant $(P=0.09$ for PD-L1, $P=0.15$ for FOXP3, and $P=0.13$ for CD163). As the levels of 
a
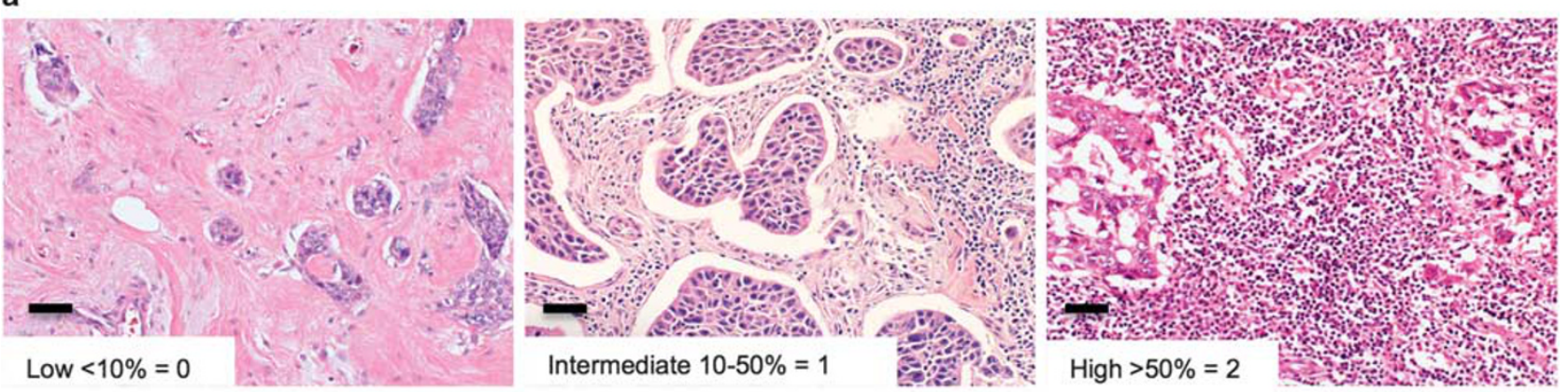

b
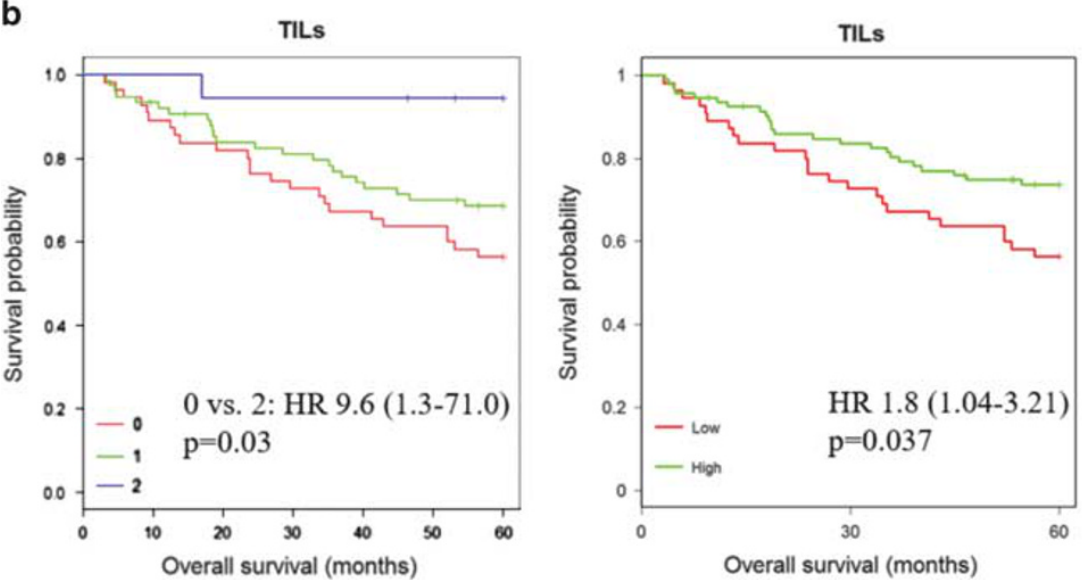

Figure 1 Tumor-infiltrating cells and prognosis. (a) Stromal tumor-infiltrating lymphocytes were evaluated on full face H\&E sections from the blocks used for tissue microarrays construction by a single surgical pathologist using established criteria. Left: representative example of low $<10 \%$ tumor-infiltrating lymphocytes, score 0 . Middle: representative example of intermediate tumor-infiltrating lymphocytes $10-$ $50 \%$, score 1. Right: representative example of high tumor-infiltrating lymphocytes $>50 \%$, score 2. (b) Left: Kaplan-Meier analysis shows improved survival in the stromal tumor-infiltrating lymphocytes score 2 group (high) versus stromal tumor-infiltrating lymphocytes score 0 group (low). Score $0(n=55)$, Score $1(n=75)$, Score $2(n=18)$. Score 0 vs 2 : HR 9.6, $P=0.03$. Score 1 vs 2 : HR $0.15, P=0.07$, Score 1 vs 0 : HR 1.5, $P=0.17$. Right: Kaplan-Meier analysis shows improved survival in the stromal tumor-infiltrating lymphocytes high group versus the stromal tumor-infiltrating lymphocytes low group. Low $=$ Score $0(n=55)$. High $=$ Score $1,2(n=93)$. HR 1.8, $P=0.037$. Scale bar size is $100 \mu \mathrm{m}$.

tumor-infiltrating lymphocytes and the immuneregulatory markers were independent, pair-wise comparisons were employed to differentiate the prognostic significance within the low tumorinfiltrating lymphocytes sub-group (Figures 3b-d). These data showed that high FOXP3 (Figure 3c) and high CD163 (Figure 3d) in a low tumor-infiltrating lymphocyte environment denote subgroups of patients with a particularly poor prognosis.

\section{Relevance of a Glycolytic Tumor Environment to Immunological Features of the Tumor}

Metabolic preference within the tumor microenvironment is known to influence the immunological milieu. ${ }^{36}$ MCT4 is critical for lactate export and its high expression defines glycolytic tumors. As shown in the representative micrographs, distinct levels of MCT4 were noted in the tumor stroma (Figure 4a). Elevated levels of stromal MCT4 staining were associated with significantly decreased overall survival (Figure 4a). To assess how stromal MCT4 expression impacts on the immune milieu, the levels of CD163, PD-L1, and FOXP3 were stratified by MCT4 levels. These analyses demonstrated that high levels of MCT4 were significantly associated with increased levels of each of the immunesuppressive markers (Figure 4b). This finding suggests that the metabolic features of the tumor microenvironment may have a pivotal role in directing the immunological features of triple negative breast cancer.

\section{Composite Analysis of Immune and Metabolic Markers Define Novel Prognostic Subtypes in Triple Negative Breast Cancer}

To concordantly analyze the immune and metabolic features of the cases, K-means clustering analysis was used to define four distinct triple negative breast cancer subtypes (Figure 5a). Cluster 1 and cluster 2 showed low levels of immunosuppressive markers PD-L1 and FOXP3, and they harbored increased tumor-infiltrating lymphocytes. By Kaplan-Meier 

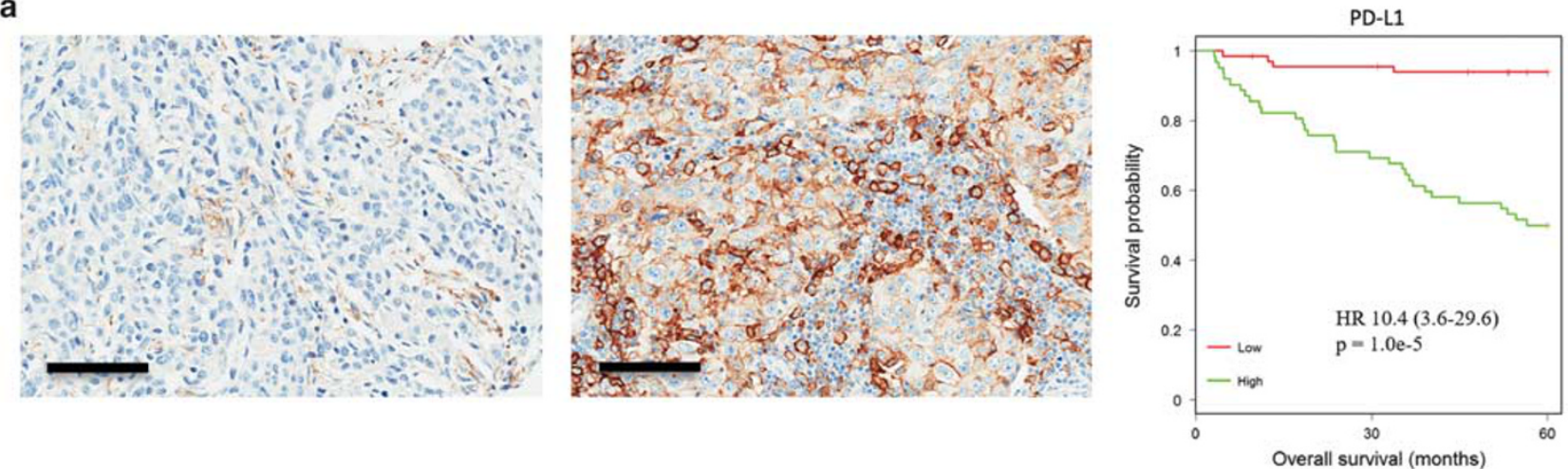

b
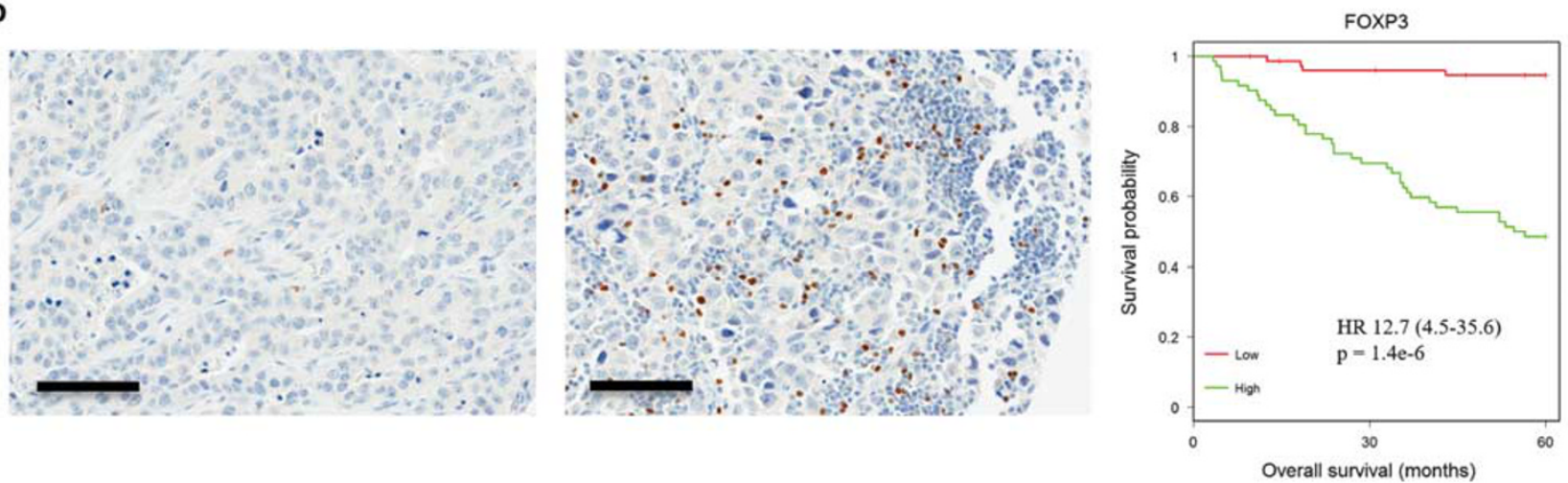

C

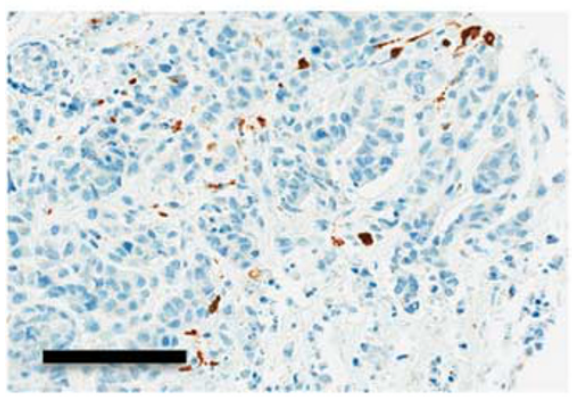

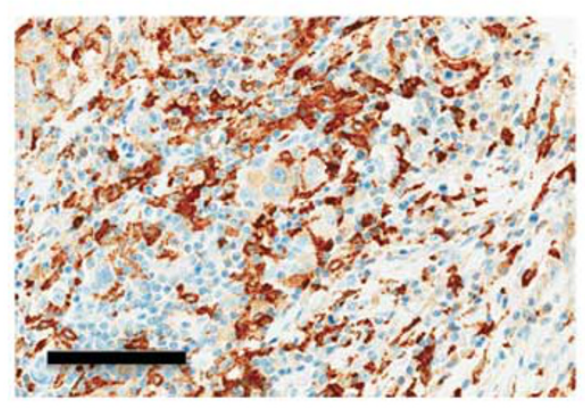

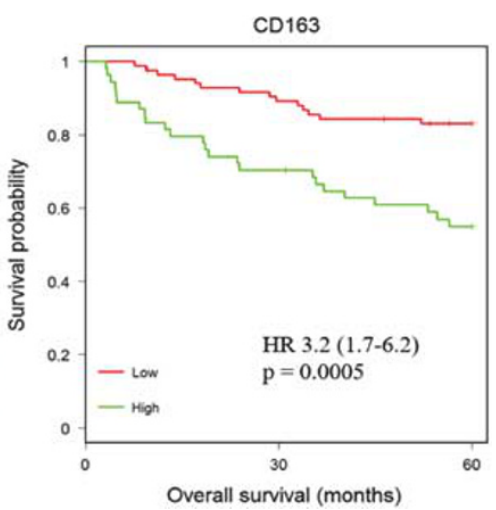

Figure 2 Immunosuppressive markers are associated with prognosis. (a) Left: PD-L1 staining by immunohistochemistry was scored in the tumor cells as low $\leq 10 \%$ in 66 cases. Middle: PD-L1 staining by immunohistochemistry was scored high $>10 \%$ in 62 cases. Right: in univariate analysis, elevated levels of PD-L1 staining were associated with significantly decreased overall survival (HR 10.4, $P=1.0 \mathrm{e}-5)$ by Kaplan-Meier analysis. (b) Left: FOXP3 staining was categorized as low, $\leq 8$, in 76 cases. Middle: High, $>8$, in 72 cases. Right: in univariate analysis, elevated levels of FOXP3 staining were associated with significantly decreased overall survival (HR 12.7, $P=1.4 \mathrm{e}-6)$ by Kaplan-Meier analysis. (c) Left: CD163 staining was noted, highlighting macrophages in the tumor cellular environment, categorized as low $\leq 20 \%$ in 84 cases. Middle: High, $>20 \%$, in 54 cases. Right: in univariate analysis, elevated levels of CD163 staining were associated with significantly decreased overall survival (HR 3.2, $P=0.0005$ ) by Kaplan-Meier analysis. Scale bar size is $100 \mu \mathrm{m}$.

analysis, these clusters had an overall good prognosis (Figure 5b). In contrast, cluster 3 and cluster 4 showed increased staining for PD-L1, FOXP3, and stromal MCT4 (Figure 5a). Both of these clusters showed poor survival (Figure 5b). Cluster 3 was enriched for CD163 macrophages with minimal tumor-infiltrating lymphocytes, whereas cluster 4 demonstrated high tumor-infiltrating lymphocytes with minimal CD163 expression (Figure 5b). These data were largely recapitulated in a sub-group analysis of patients for whom we had adjuvant chemotherapy treatment information. (Supplementary Figure 1). In univariate analysis the clusters were significantly associated with overall survival, both including all clusters (logrank) or in pair-wise comparison (clusters 1, 2 vs 3, 4). In multivariate analysis, the K-means cluster group determined by combined marker expression showed strong prognostic significance, with a hazard ratio 


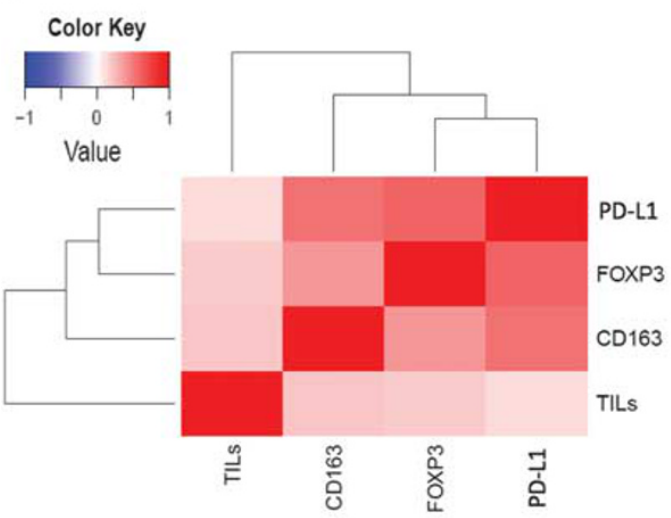

C

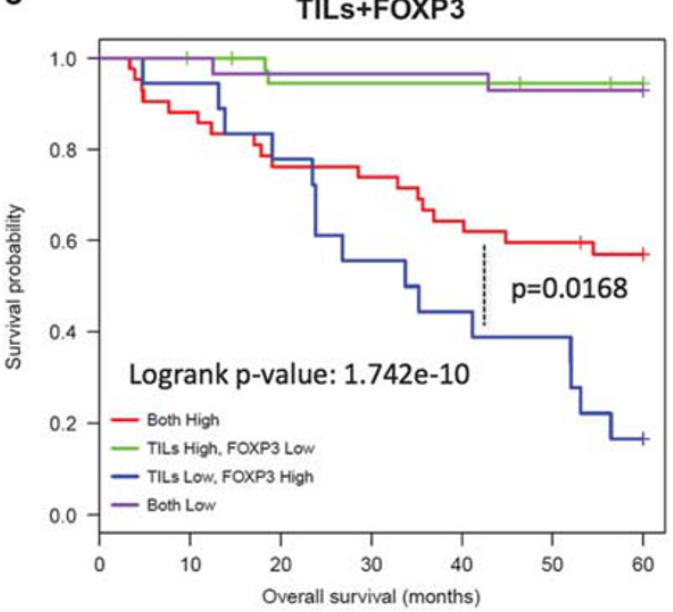

b

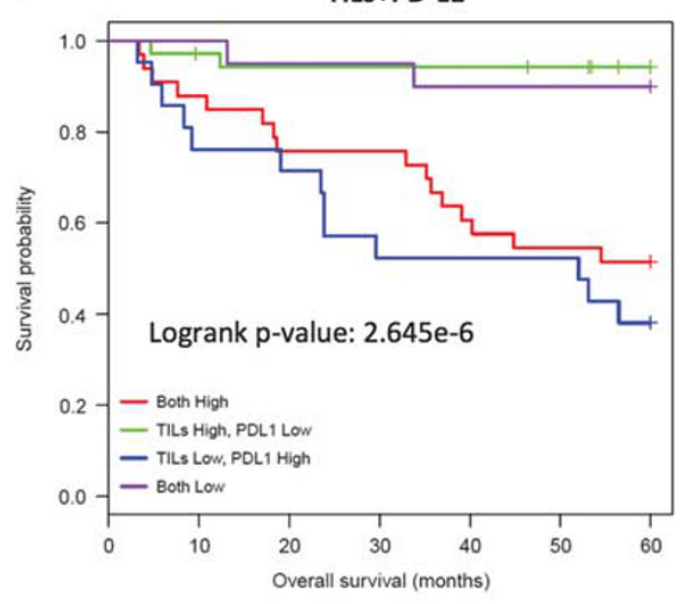

d

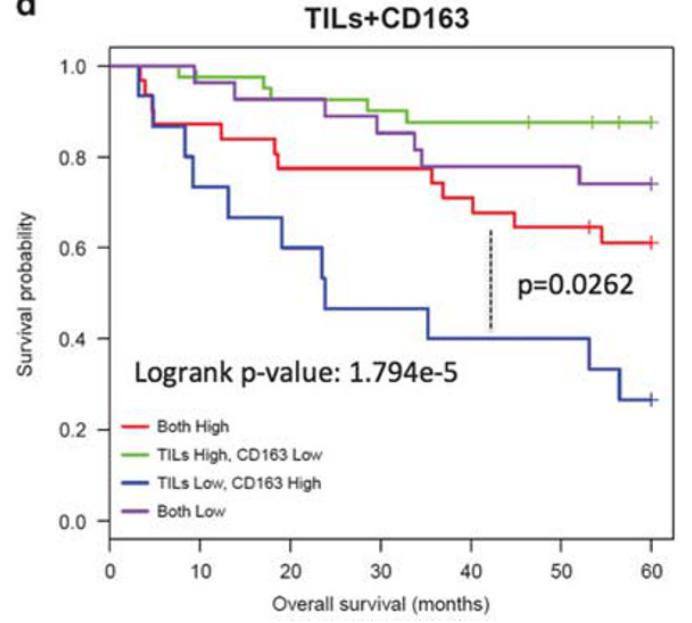

Figure 3 Correlation between multiple immune-suppressive mechanisms in triple negative breast cancer. (a) Heatmap showing the Spearman correlation between the indicated markers. The association between CD163, FOXP3, and PD-L1 are all significant $(P<0.001)$. (b) Composite Kaplan-Meier analysis of the levels of tumor-infiltrating lymphocytes and PD-L1. (c) Composite Kaplan-Meier analysis of the levels of tumor-infiltrating lymphocytes and FOXP3. (d) Composite Kaplan-Meier analysis of the levels of tumor-infiltrating lymphocytes and CD163.

greater than for tumor stage and histologic grade (Figure 5c). Together, these data suggest that composite analysis of immunological and metabolic determinants of the tumor microenvironment could define important subgroups of triple negative breast cancer.

\section{Discussion}

In this study, we investigated the expression of immune markers PD-L1, FOXP3, CD163, and tumorinfiltrating lymphocytes, as well as the metabolic marker MCT4 in a cohort of triple negative breast cancer patients.

The finding of improved survival with high levels of stromal tumor-infiltrating lymphocytes is consistent with other studies in triple negative breast cancer. Tumor-infiltrating lymphocytes have generally been associated with better prognosis and response to neoadjuvant therapy in triple negative breast cancer. ${ }^{35,37-42}$ In recent meta-analysis studies higher tumor-infiltrating lymphocyte levels in pretreatment biopsy indicated higher pathologic complete response rates for neoadjuvant chemotherapy and improved survival ${ }^{43,44}$ Therefore, the enumeration of tumor-infiltrating lymphocytes can be both a predictive marker of response to chemotherapy and prognostic marker in triple negative breast cancer. We focused on stromal tumor-infiltrating lymphocytes in our analysis as suggested by the International Tumor-Infiltrating Lymphocytes Working Group; ${ }^{18}$ however, others have also shown prognostic significance of tumor-infiltrating lymphocytes within the epithelial compartment. ${ }^{45}$

As tumor-infiltrating lymphocytes represent a complex population of lymphocytes, there is considerable interest in defining the immunological features of tumor-infiltrating lymphocytes. Here, we employed FOXP3 as a marker for regulatory $\mathrm{T}$ cells and CD163 as a marker of type 2 macrophages. These 

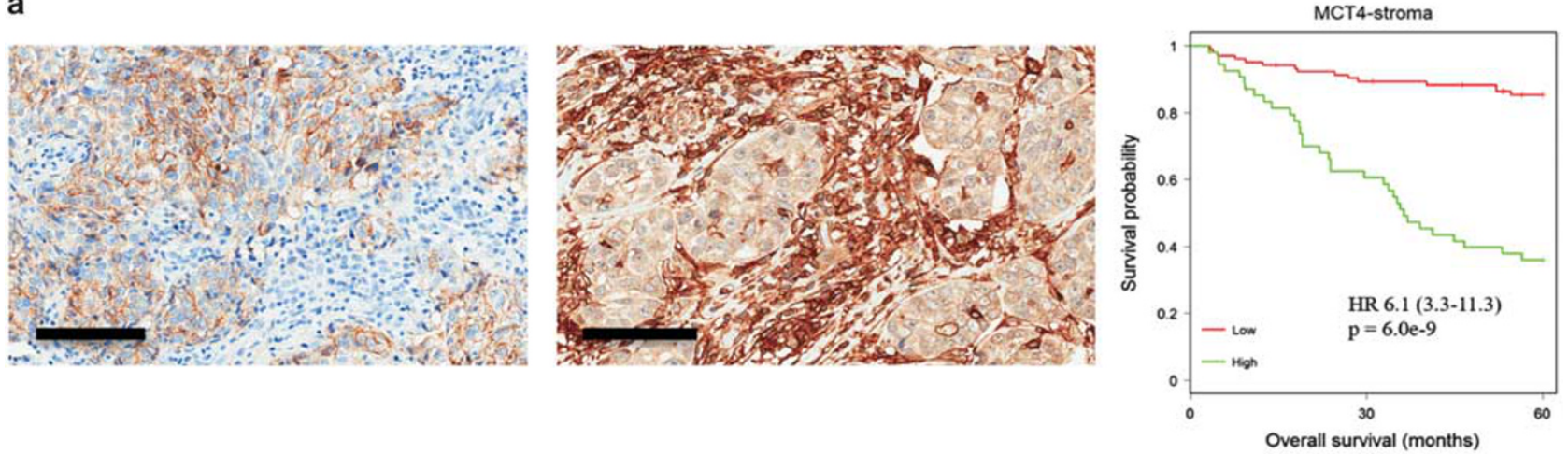

b

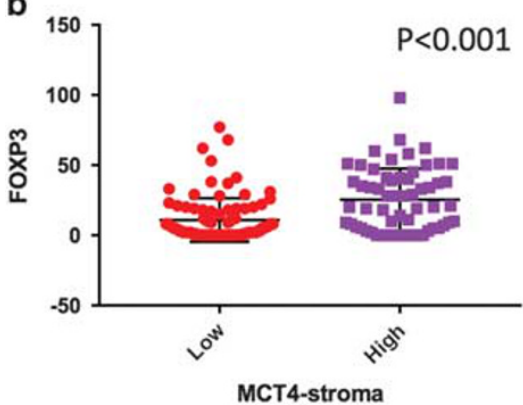

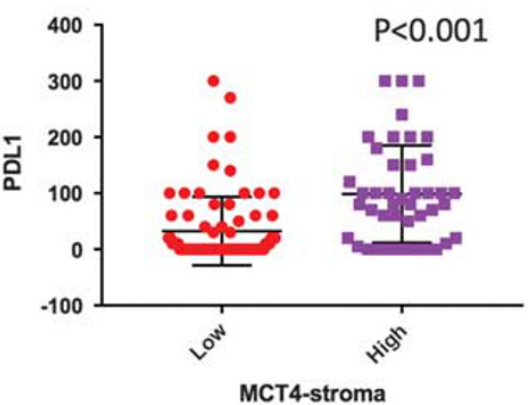

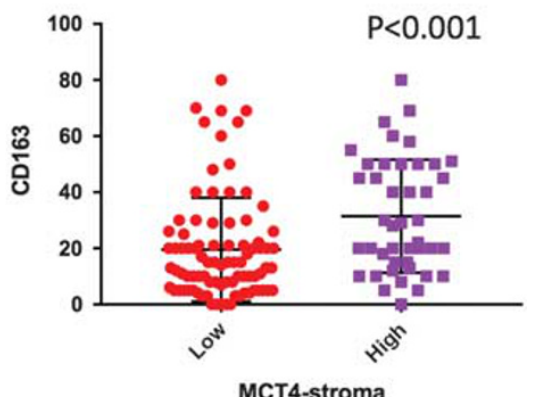

Figure 4 Metabolic features of the tumor environment are associated with prognosis and immune-suppressive markers. (a) Left and middle: stromal MCT4 staining was evaluated by immunohistochemistry, and categorized as low (score 0,1) vs high (score 2). Right: in univariate analysis, elevated levels of stromal MCT4 staining were associated with significantly decreased overall survival (HR 6.1, $P=6.0$ e-9) by Kaplan-Meier analysis. (b) The level of MCT4 in the stroma was used to dichotomize cases and the levels of CD163, FOXP3, and PD-L1-positive cells is shown. Statistical analysis is by $t$-test. Scale bar size is $100 \mu \mathrm{m}$.

immune cells are generally immunosuppressive, and are believed to contribute to immune evasion by tumor cells. Regulatory T cells can suppress proliferation and cytokine secretion by effector $\mathrm{T}$ lymphocytes and could contribute to overall tumorinfiltrating lymphocyte density within a tumor. ${ }^{24}$ CD163-expressing macrophages are alternatively activated macrophages that enhance tumor progression by promoting tumor cell invasion, migration, and angiogenesis. ${ }^{25}$ Elevated levels of regulatory T-cell lymphocytes and CD163-expressing macrophages were associated with decreased survival in this study and there was a significant correlation between the presence of regulatory $\mathrm{T}$ cells and CD163-positive macrophages. These findings suggest that features of a specific tumor and/or its environment elicit multiple mechanisms of local immune suppression.

There is considerable interest in understanding the nature of immunosuppressive mechanisms in triple negative breast cancer that could be specifically targeted to yield effective treatment. PD-L1 is a target of the therapeutic antibody Nivolumab that is FDA approved for specific cancer indications. In our study PD-L1 levels were strongly associated with shorter overall survival. While there have been conflicting reports regarding the impact on PD-L1 expression on prognosis, several studies showed decreased survival in cases with high PD-L1 staining as evaluated by immunohistochemistry, concordant with our study. For example, in a study of 650 cases of breast cancer, PD-L1 was expressed in $23.4 \%$ of cases and was associated with worse overall survival. ${ }^{46}$ Similarly, a recent meta-analysis of PD-L1 in breast carcinoma, indicated that PD-L1 expression was marker of poor prognosis. ${ }^{47}$ As PDL1 is expressed in a significant percentage of triple negative breast cancer, it represents a potential therapeutic target, ${ }^{20}$ and numerous phase I/II clinical trials testing antibodies against PD-1 or PD-L1 are ongoing. ${ }^{48}$ Interestingly, the PD-L1 gene (CD274) is amplified in a significant subset of triple negative breast cancer cases. However, in analysis of data from TCGA this event did not yield a significant increase in transcript expression, suggesting nongenetic regulation of PD-L1 expression. Therefore, direct analysis of the protein may be a preferable determinant of PD-L1 function within the tumor compartment. In many triple negative breast cancer cases, PD-L1 expression was associated with the presence of CD163-positive macrophages and FOXP3-positive regulatory $\mathrm{T}$ cells. This finding suggests that coordinate targeting of multiple immunological features of triple negative breast cancer may be required to mount an effective antitumor response. Consistent with this concept, it has been suggested that a combined strategy to block PD-L1/ PD-1 along with modalities to deplete regulatory 
a

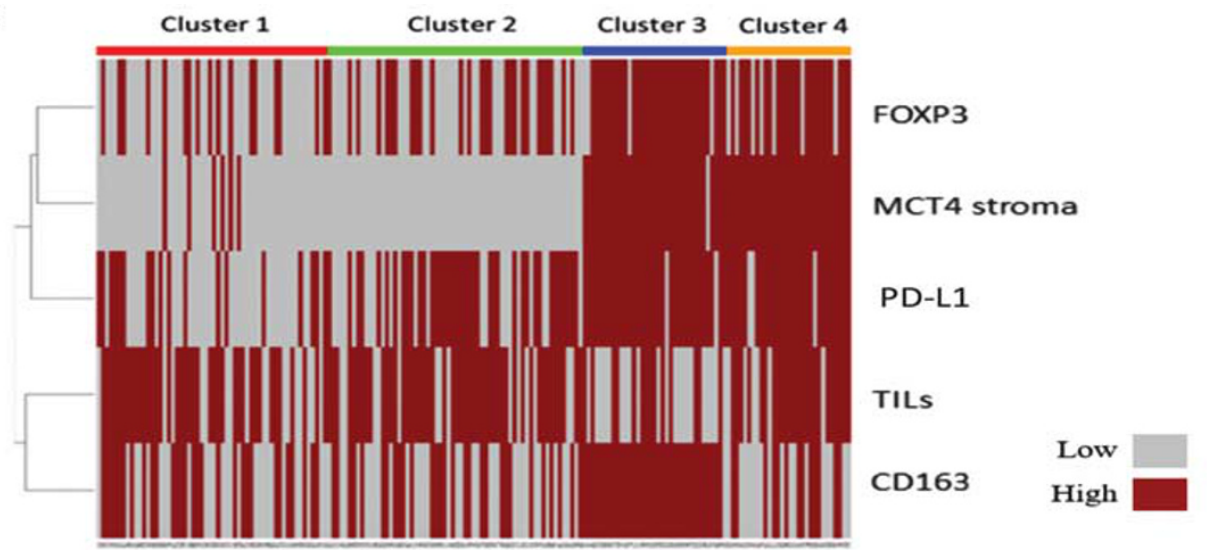

b

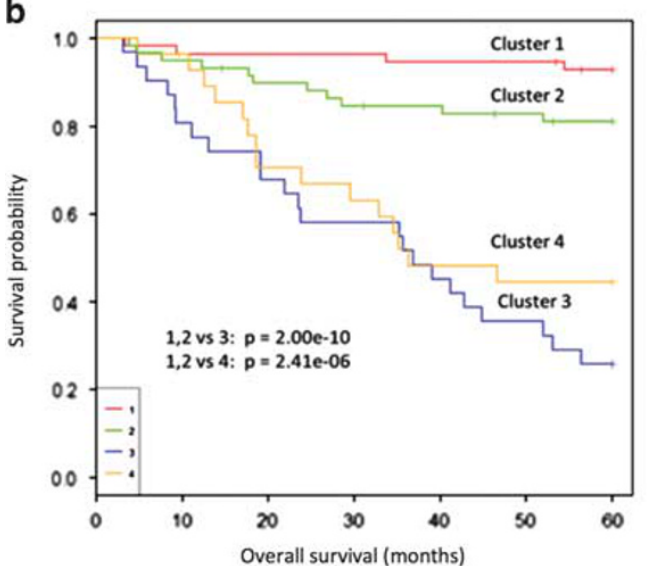

C

\begin{tabular}{c|c|c|c} 
Univariate & HR & Cl 95\% & p-value (logrank) \\
\hline \hline Cluster & 2.1436 & $1.667-2.756$ & $1.443 \mathrm{e}-10$ \\
Cluster 1,2 vs 3,4 & 7.0271 & $3.849-12.83$ & $1.911 \mathrm{e}-13$ \\
TILs (2 groups) & 0.5475 & $0.3108-0.9643$ & 0.03421 \\
TILs (3 groups) & 0.5240 & $0.3286-0.8356$ & 0.005863 \\
Tumor Stage & 1.4137 & $0.9755-2.049$ & 0.0674 \\
Histology Grade & 1.8362 & $0.8983-3.753$ & 0.0957 \\
LN Status & 3.539 & $1.8-6.96$ & $9.266 \mathrm{e}-05$ \\
& & & \\
Multivariate & $\mathrm{HR}$ & $\mathrm{Cl} 95 \%$ & $\mathrm{p}$-value \\
\hline \hline Cluster 1,2 vs 3,4 & 7.5689 & $3.6146-15.849$ & $7.97 \mathrm{e}-08$ \\
Tumor Stage & 1.4803 & $0.8697-2.520$ & 0.148 \\
Histology Grade & 1.7720 & $0.7816-4.017$ & 0.171 \\
LN Status & 4.0924 & $2.0275-8.260$ & $8.41 \mathrm{e}-05$
\end{tabular}

Figure 5 Composite analysis of immune and metabolic markers. (a) K-means clustering analysis plot that defines four distinct triple negative breast cancer subtypes. (b) Kaplan-Meier plot indicating survival probability based on the indicated clusters. Note decreased survival in cluster 3 and 4 . Cluster 3 vs 1, 2: HR 8.38, $P=2.00 \mathrm{e}-10$. Cluster 4 vs 1, 2: HR 5.64, $P=2.41 \mathrm{e}-6$. Cluster $1: n=56$. Cluster 2: $n=59$. Cluster 3: $n=31$. Cluster 4: $n=28$. (c) Univariate and multivariate analysis of the $\mathrm{K}$-means clusters, tumor stage, grade, and lymph node status. The clusters identified based on composite analysis of immune and metabolic markers maintain statistical significance $(P<0.05)$ in the multivariate model.

$\mathrm{T}$ cells may be a reasonable therapeutic option. ${ }^{24}$ Similarly, there are ongoing approaches to selectively target tumor-associated macrophages. ${ }^{49,50}$

The cancer microenvironment was postulated to modulate immune cell infiltration and immune cell function. ${ }^{36,51,52}$ Interestingly, triple negative breast cancer tumors analyzed in this study showed strong correlation between distinct immunosuppressive features. This finding supports the hypothesis that the tumor niche yields a multi-factorial impact on the immune system. Glycolytic metabolic preference of the tumor microenvironment represents one of the potential mechanisms promoting immunosuppressive infiltrate. ${ }^{53,54}$ Glycolytic tumors express transporters such as MCT4 that facilitate extracellular removal of lactate to support metabolism. This has the net effect of acidifying the local tumor environment. ${ }^{55}$ Multiple studies have shown that MCT4 expression in the tumor microenvironment is associated with poor prognosis. ${ }^{56,57}$ How MCT4 impacts on the local immunological milieu remains poorly understood. However, lactic acid can skew macrophages toward the anti-inflammatory tumor- promoting M2 phenotype. ${ }^{58}$ This may represent a possible mechanistic link for the relationship between the metabolic and immune environment. Heretofore the relationship of MCT4 with PD-L1 and FOXP3 in triple negative breast cancer has not been determined, but findings from our study suggest that metabolic features of the tumor environment could have a broad influence on the composition of immune infiltrate and related therapeutic sensitivities.

Given the complexity of tumor biology, it is increasingly important to consider the intersection of different processes in delineating tumor subtypes. Integrating the data in this study defined four subtypes of triple negative breast cancer based on their relative tumor-infiltrating lymphocyte, immune-suppressive features, and MCT4 status. The subsets identified in this manner may have important implications for the use of immunotherapy in the treatment of triple negative breast cancer. Cluster 1 had high levels of tumor-infiltrating lymphocytes, but relatively low levels of PD-L1 and FOXP3. This suggests that potentially inhibiting 
CTLA4 or other approaches to activate the immune system could be effective in this subtype. Cluster 2 harbored high levels of tumor-infiltrating lymphocytes, but had high levels of PD-L1 with relatively scant presentation of FOXP3- and CD163-positive cells, suggesting the utility of agents inhibiting PD-1/ PD-L1. Interestingly, the two clusters had a more favorable prognosis in the cohort analyzed. Clusters 3 and 4 had poor prognosis and were characterized by a glycolytic environment and the presence of multiple immunosuppressive features. Further studies will be required to determine whether the association between MCT4 and the nature of the immune system is causal or casual. As overall survival is typically associated with metastatic spread, it will also be critically important to evaluate how the markers studied herein directly contribute to the invasive potential and ability to colonize at distant sites. As cluster 3 was largely devoid of tumor-infiltrating lymphocytes it would require therapies to engage lymphocytes (eg, through vaccine) that would couple with targeting regulatory $\mathrm{T}$ cells and tumor-associated macrophages. Cluster 4 showed high tumor-infiltrating lymphocytes with minimal CD163 expressing macrophages, suggesting that immune checkpoint therapy could be effective in this subtype in conjunction with suppression of regulatory T cells. Thus, the defined subgroups have unique features that may present opportunities for targeted therapy, and suggest that a coordinate assessment of immunological features may be important for effective use of immunotherapy in triple negative breast cancer. Furthermore, functional research focused on interrelated immune and metabolic pathways could be fruitful in increasing the breadth of antitumor immunologic responses.

\section{Acknowledgments}

This work was supported by NIH grants to AKW (CA163863) and ESK (CA188650).

\section{Disclosure/conflict of interest}

The authors declare no conflict of interest.

\section{References}

1 Badve S, Dabbs DJ, Schnitt SJ, et al. Basal-like and triple-negative breast cancers: a critical review with an emphasis on the implications for pathologists and oncologists. Mod Pathol 2011;24:157-167.

2 Haffty BG, Yang Q, Reiss M, et al. Locoregional relapse and distant metastasis in conservatively managed triple negative early-stage breast cancer. J Clin Oncol 2006;24: 5652-5657.

3 Reis-Filho JS, Tutt AN. Triple negative tumours: a critical review. Histopathology 2008;52:108-118.

4 Thike AA, Cheok PY, Jara-Lazaro AR, et al. Triplenegative breast cancer: clinicopathological characteristics and relationship with basal-like breast cancer. Mod Pathol 2010;23:123-133.

5 Dent R, Trudeau M, Pritchard KI, et al. Triple-negative breast cancer: clinical features and patterns of recurrence. Clin Cancer Res 2007;13:4429-4434.

6 Bauer KR, Brown M, Cress RD, Parise CA, Caggiano V. Descriptive analysis of estrogen receptor (ER)-negative, progesterone receptor (PR)-negative, and HER2negative invasive breast cancer, the so-called triplenegative phenotype: a population-based study from the California cancer Registry. Cancer 2007;109: 1721-1728.

7 Harris LN, Broadwater G, Lin NU, et al. Molecular subtypes of breast cancer in relation to paclitaxel response and outcomes in women with metastatic disease: results from CALGB 9342. Breast Cancer Res 2006;8:R66.

8 Morris GJ, Naidu S, Topham AK, et al. Differences in breast carcinoma characteristics in newly diagnosed African-American and Caucasian patients: a singleinstitution compilation compared with the National Cancer Institute's Surveillance, Epidemiology, and End Results database. Cancer 2007;110:876-884.

9 Li X, Wetherilt CS, Krishnamurti U, et al. Stromal PDL1 Expression Is Associated With Better Disease-Free Survival in Triple-Negative Breast Cancer. Am J Clin Pathol 2016;146:496-502.

10 Millar EK, Graham PH, O'Toole SA, et al. Prediction of local recurrence, distant metastases, and death after breast-conserving therapy in early-stage invasive breast cancer using a five-biomarker panel. J Clin Oncol 2009;27:4701-4708.

11 Yam C, Mani SA, Moulder SL. Targeting the molecular subtypes of triple negative breast cancer: understanding the diversity to progress the field. Oncologist 2017;22:1086-1093.

12 Swisher SK, Wu Y, Castaneda CA, et al. Interobserver agreement between pathologists assessing tumorinfiltrating lymphocytes (TILs) in breast cancer using methodology proposed by the international TILs working group. Annals of Surgical Oncology 2016;23: 2242-2248.

13 Garcia-Teijido P, Cabal ML, Fernandez IP, Perez YF. Tumor-infiltrating lymphocytes in triple negative breast cancer: the future of imune targeting. Clin Med Insights Oncol 2016;10:31-39.

14 Stanton SE, Disis ML. Clinical significance of tumorinfiltrating lymphocytes in breast cancer. J Immunother Cancer 2016;4:59.

15 Page DB, Postow MA, Callahan MK, Allison JP, Wolchok JD. Immune modulation in cancer with antibodies. Annu Rev Med 2014;65:185-202.

16 Peggs KS, Quezada SA, Korman AJ, Allison JP. Principles and use of anti-CTLA4 antibody in human cancer immunotherapy. Curr Opin Immunol 2006;18: 206-213.

17 Luen SJ, Savas P, Fox SB, Salgado R, Loi S. Tumourinfiltrating lymphocytes and the emerging role of immunotherapy in breast cancer. Pathology 2017;49: 141-155.

18 Salgado R, Denkert C, Demaria S, et al. The evaluation of tumor-infiltrating lymphocytes (TILs) in breast cancer: recommendations by an International TILs Working Group 2014. Ann Oncol 2015;26:259-271.

19 Boussiotis VA. Molecular and biochemical aspects of the PD-1 checkpoint pathway. N Engl J Med 2016;375: 1767-1778. 
20 Mittendorf EA, Philips AV, Meric-Bernstam F, et al. PD-L1 expression in triple-negative breast cancer. Cancer Immunol Res 2014;2:361-370.

21 Disis ML, Stanton SE. Triple-negative breast cancer: immune modulation as the new treatment paradigm. Am Soc Clin Oncol Educ Book 2015;e25-e30.

22 Nanda R, Chow LQ, Dees EC, et al. Pembrolizumab in patients with advanced triple-negative breast cancer: phase Ib KEYNOTE-012 study. J Clin Oncol 2016;34: 2460-2467.

23 Cimino-Mathews A, Foote JB, Emens LA. Immune targeting in breast cancer. Oncology (Williston Park, NY) 2015;29:375-385.

$24 \mathrm{Li} \mathrm{Z}$, Dong P, Ren M, et al. PD-L1 Expression Is Associated with Tumor FOXP3(+) Regulatory T-Cell Infiltration of Breast Cancer and Poor Prognosis of Patient. J Cancer 2016;7:784-793.

25 Medrek C, Ponten F, Jirstrom K, Leandersson K. The presence of tumor associated macrophages in tumor stroma as a prognostic marker for breast cancer patients. BMC Cancer 2012;12:306.

26 Bobisse S, Foukas PG, Coukos G, Harari A. Neoantigenbased cancer immunotherapy. Ann Transl Med 2016; $4: 262$.

27 Shigehisa Kitano AI. Cancer neoantigens: a promising source of immunogens for cancer immunotherapy. J Clin Cell Immunology 2015;06:2-7.

28 Hutcheson J, Balaji U, Porembka MR, et al. Immunologic and metabolic features of pancreatic ductal adenocarcinoma define prognostic subtypes of disease. Clin Cancer Res 2016;22:3606-3617.

29 Doyen J, Trastour C, Ettore F, et al. Expression of the hypoxia-inducible monocarboxylate transporter MCT4 is increased in triple negative breast cancer and correlates independently with clinical outcome. Biochem Biophys Res Commun 2014;451:54-61.

30 Supuran CT. Carbonic anhydrases: novel therapeutic applications for inhibitors and activators. Nat Rev Drug Discov 2008;7:168-181.

31 Halestrap AP. The monocarboxylate transporter familyStructure and functional characterization. IUBMB Life 2012;64:1-9.

32 Witkiewicz AK, Whitaker-Menezes D, Dasgupta A, et al. Using the "reverse Warburg effect" to identify high-risk breast cancer patients: stromal MCT4 predicts poor clinical outcome in triple-negative breast cancers. Cell Cycle 2012;11:1108-1117.

33 Knudsen E, Vail P, Balaji U et al. Stratification of pancreatic ductal adenocarcinoma: combinatorial genetic, stromal, and immunological markers. Clin Cancer Res 2017;23:4429-4440.

34 R_Core_Team. R: A Language and environment for statistical computing. R Foundation For Statistical Computing: Vienna, Austria, 2014.

35 Loi S, Michiels S, Salgado R, et al. Tumor infiltrating lymphocytes are prognostic in triple negative breast cancer and predictive for trastuzumab benefit in early breast cancer: results from the FinHER trial. Ann Oncol 2014;25:1544-1550.

36 Pilon-Thomas S, Kodumudi KN, El-Kenawi AE, et al. Neutralization of tumor acidity improves antitumor responses to immunotherapy. Cancer Res 2016;76: 1381-1390.

37 Pruneri G, Vingiani A, Bagnardi V, et al. Clinical validity of tumor-infiltrating lymphocytes analysis in patients with triple-negative breast cancer. Ann Oncol 2016;27:249-256.
38 Perez EA, Ballman KV, Tenner KS, et al. Association of stromal tumor-infiltrating lymphocytes with recurrence-free survival in the N9831 adjuvant trial in patients with early-stage HER2-positive breast cancer. JAMA Oncol 2016;2:56-64.

39 Ali HR, Provenzano E, Dawson SJ, et al. Association between CD8+ T-cell infiltration and breast cancer survival in 12,439 patients. Ann Oncol 2014;25: 1536-1543.

40 Dieci MV, Mathieu MC, Guarneri V, et al. Prognostic and predictive value of tumor-infiltrating lymphocytes in two phase III randomized adjuvant breast cancer trials. Ann Oncol 2015;26:1698-1704.

41 Loi S, Sirtaine N, Piette F, et al. Prognostic and predictive value of tumor-infiltrating lymphocytes in a phase III randomized adjuvant breast cancer trial in node-positive breast cancer comparing the addition of docetaxel to doxorubicin with doxorubicin-based chemotherapy: BIG 02-98. J Clin Oncol 2013;31: 860-867.

42 Dieci MV, Criscitiello C, Goubar A, et al. Prognostic value of tumor-infiltrating lymphocytes on residual disease after primary chemotherapy for triple-negative breast cancer: a retrospective multicenter study. Ann Oncol 2014;25:611-618.

43 Mao Y, Qu Q, Zhang $\mathrm{Y}$, et al. The value of tumor infiltrating lymphocytes (TILs) for predicting response to neoadjuvant chemotherapy in breast cancer: a systematic review and meta-analysis. PLoS ONE 2014;9: e115103.

44 Wang K, Xu J, Zhang T, Xue D. Tumor-infiltrating lymphocytes in breast cancer predict the response to chemotherapy and survival outcome: a meta-analysis. Oncotarget 2016;7:44288-44298.

45 Krishnamurti U, Wetherilt CS, Yang J, Peng L, Li X. Tumor-infiltrating lymphocytes are significantly associated with better overall survival and diseasefree survival in triple-negative but not estrogen receptor-positive breast cancers. Hum Pathol 2017;64: 7-12.

46 Muenst S, ARS, Gao F, et al. Expression of programmed death ligand 1 (PD-L1) is associated with poor prognosis in human breast cancer. Breast Cancer Res Treat 2014;146:15-24.

47 Yawen Guo PY, Liu Zeming, et al. Prognostic and clinicopathological value of programmed death ligand1 in breast cancer: a meta-analysis. PLoS ONE 2016;11: e0156323.

$48 \mathrm{Li}$ X, Li M, Lian Z, et al. Prognostic role of programmed death ligand-1 expression in breast cancer: a systematic review and meta-analysis. Targ Oncol 2016;11: 753-761.

49 Condeelis J, Pollard JW. Macrophages: obligate partners for tumor cell migration, invasion, and metastasis. Cell 2006;124:263-266.

50 Mitchem JB, Brennan DJ, Knolhoff BL, et al. Targeting tumor-infiltrating macrophages decreases tumor-initiating cells, relieves immunosuppression, and improves chemotherapeutic responses. Cancer Res 2013;73:1128-1141.

51 Marme F. Immunotherapy in breast cancer. Oncol Res Treat 2016;39:335-345.

52 Schlosser HA, Theurich S, Shimabukuro-Vornhagen A, et al. Overcoming tumor-mediated immunosuppression. Immunotherapy 2014;6:973-988.

53 Singer K, Kastenberger M, Gottfried E, et al. Warburg phenotype in renal cell carcinoma: high expression of glucose-transporter 1 (GLUT-1) correlates with low 
CD8(+) T-cell infiltration in the tumor. Int J Cancer 2011;128:2085-2095.

54 Renner K, Singer K, Koehl GE, et al. Metabolic hallmarks of tumor and immune cells in the tumor microenvironment. Front Immunol 2017;8:248.

55 Gillies RJ, Gatenby RA. Metabolism and its sequelae in cancer evolution and therapy. Cancer J 2015;21: 88-96.

56 Baek G, Tse YF, Hu Z, et al. MCT4 defines a glycolytic subtype of pancreatic cancer with poor prognosis and unique metabolic dependencies. Cell reports 2014;9: 2233-2249.

57 Le Floch R, Chiche J, Marchiq I, et al. CD147 subunit of lactate/H+ symporters MCT1 and hypoxia-inducible MCT4 is critical for energetics and growth of glycolytic tumors. Proc Natl Acad Sci USA 2011;108: 16663-16668.

58 Colegio OR, Chu NQ, Szabo AL, et al. Functional polarization of tumour-associated macrophages by tumour-derived lactic acid. Nature 2014;513:559-563.

Supplementary Information accompanies the paper on Modern Pathology website (http://www.nature.com/ modpathol) 